\title{
Prevalence and evolution of drug resistance HIV-1 variants in Henan, China
}

\author{
Jing Yun $\mathrm{LI}^{1, *}$, Han Ping $\mathrm{LI}^{1}$, Lin $\mathrm{LI}^{1}$, Hong $\mathrm{LI}^{2}$, Zhe WANG ${ }^{2}$, Kun YANG ${ }^{1}$, Zuo Yi BAO ${ }^{1}$, Dao Min ZHUANG ${ }^{1}, \mathrm{Si}$ \\ Yang LIU ${ }^{1}$, Yong Jian $\mathrm{LIU}^{1}$, Hui XING ${ }^{3}$, Yi Ming SHAO ${ }^{3}$ \\ ${ }^{1}$ AIDS Research Department, Institute of Microbiology and Epidemiology, Academy of Military Medical Science, Beijing \\ 100071, China \\ ${ }^{2}$ Center of Disease Prevention and Control, Henan Province, China \\ ${ }^{3}$ National Center for AIDS/STD Control and Prevention, China CDC, Beijing, China
}

\begin{abstract}
ABSTACT
To understand the prevalence and evolution of drug resistant HIV strains in Henan China after the implementation of free antiretroviral therapy for AIDS patients. 45 drug naïve AIDS patients, 118 AIDS patients who received three months antiretroviral therapy and 124 AIDS patients who received six months antiretroviral treatment were recruited in the southern part of Henan province. Information on general condition, antiretroviral medicines, adherence and clinical syndromes were collected by face to face interview. Meanwhile, $14 \mathrm{ml}$ EDTA anticoagulant blood was drawn. CD4/CD8 $\mathrm{T}$ cell count, viral load and genotypic drug resistance were tested. The rates of clinical improvement were $55.1 \%$ and $50.8 \%$ respectively three months and six months after antiretroviral therapy. The mean CD4 cell count after antiretroviral therapy was significantly higher than in drug naïve patients. The prevalence rate of drug resistant HIV strains were 13 . $9 \%, 45.4 \%$ and $62.7 \%$ in drug naïve patients, three month treatment patients and six month treatment patients, respectively. The number of resistance mutation codons and the frequency of mutations increased significantly with continued antiretroviral therapy. The mutation sites were primarily at the 103, 106 and 215 codons in the three-month treatment group and they increased to 15 codon mutations in the six-month treatment group. From this result, the evolution of drug resistant strains was inferred to begin with the high level NNRTI resistant strain, and then develop low level resistant strains to NRTIs. The HIV strains with high level resistance to NVP and low level resistance to AZT and DDI were highly prevalent because of the AZT+DDI+NVP combination therapy. These HIV strains were also cross resistant to DLV, EFV, DDC and D4T. Poor adherence to therapy was believed to be the main reason for the emergence and prevalence of drug resistant HIV strains. The prevalence of drug resistant HIV strains was increased with the continuation of antiretroviral therapy in the southern part of Henan province. Measures, that could promote high level adherence, provide new drugs and change ART regimens in failing patients, should be implemented as soon as possible.
\end{abstract}

Keywords: AIDS, drug resistance, adherence, antiretroviral therapy.

\section{INTRODUCTION}

Highly Active Antiretroviral Therapy (HAART) has significantly reduced the rate of HIV and AIDS-related morbidity and mortality. There are 21 antiretroviral drugs and over 25 formulations approved by the US Food and Drug Administration (FDA) for the treatment of HIV infected persons including 7 nucleotide and nucleoside reverse transcriptase inhibitors (NRTIs), 3 nonnucleoside reverse transcriptase inhibitors (NNRTIs), 9 protease inhibitors (PIs)

*Correspondence: Jing Yun LI

E-mail: lijy@nic.bmi.ac.cn and 1 fusion inhibitor (EIs). Through combining drugs targeting different HIV-1 replication steps, HAART delays clinical progression by suppressing viral replication, measured by a substantial reduction in HIV RNA, and allowing immune reconstitution, measured in most studies by increasing in CD4 cells count.

The relatively low fidelity and the absence of proofreading of HIV-1 reverse transcriptase (RT) lead to genetic variability of the virus [1]. Furthermore, the high rate of HIV-1 replication, the accumulation of archived proviral variants during the course of HIV-1 infection, and genetic recombination when viruses of different sequence infect the same cell enhance the viral gene variability. As a 
result, innumerable genetically distinct variants (quasispecies) evolve within an individual in the months following primary infection [2]. When treated with drugs, the viral variants are under the drugs' selective pressure; the variants with drug-resistant mutations replicate quickly and become predominant variants under this selective pressure. Consequently, there is rebound of viral replication. It is suggested that the drug resistance variants can replace the wild type variants completely within 14-28 d of treatments [3].

With the use of different antiretroviral drugs, drug-resistant HIV-1 variants are prevalent worldwide. In America and Europe, about $10 \%$ of recent HIV-1 infections are drug resistant HIV-1 strains [4]. In Canada, the prevalence of drug-resistant HIV-1 strains among recent infected individuals increased from $5.5 \%$ at $1995-1998$ to $18.5 \%$ at $1999-2000$ and $27.6 \%$ at 2001 . Now the prevalent rates of HIV drug-resistant strains are high in many countries. In 2001 , the rate is $5-11 \%$ in Switzerland, $10-17 \%$ in French, $13 \%$ in German, 14\% in England, 23-26\% in Spain, 15.4\% in Argentina [5-7]. What is more, resistant strains have been reported for almost all antiretroviral drugs. With the transmission of drug resistant HIV-1 strains, more and more multi-drug-resistant variants are reported. Now, drug resistance is one of the major reasons for antiretroviral therapy failure $[8,9]$ and also the great challenge of AIDS treatment worldwide.

In China, about 16000 AIDS patients are now treated free under the implement of the policy of "Four Free One Care". Most AIDS patients live in rural areas with limited health services, and the local physicians are inexperienced. As a result, drug-resistant HIV-1 strains will spread. So surveillance for drug-resistant HIV-1 strains is necessary to monitor the prevalence of drug-resistant HIV-1 variants, clarify the factors related to drug resistance emergence, make the policy and program of the development and application of antiretroviral drugs fruitful, and ensure the success of antiretroviral therapy. In this study, we carried out clinical and molecular epidemiological research on the prevalence HIV-1 drug resistance in Henan province, the first region implementing free antiretroviral therapy in China.

\section{MATERIALS AND METHODS}

\section{Studypopulation}

The patients recruited into the study came from Shenqiu, Henan province of China. The patients were treated with AZT + DDI + NVP since November 2003. The drugs were provided free by the local disease prevention and control branch, and the administration of the drugs was supervised by the local rural physicians. According to the time of treatment (three or six months), patients were recruited, respectively, into two cross-sectional studies. After signing informed consent, all patients were interviewed face to face by trained and qualified staffs with identical questionnaire. Information about general conditions, transmission route, clinical syndromes within three months, the antiretroviral therapy regimen, the sources of drugs, the reason for drug withdrawal, the willingness to receive therapy, the attitude toward therapy, and the history of anti-retroviral therapy, was collected. The information about adherence was collected by specified questions about the kinds and times of drug withdrawal in the past three days and in the past one month, and the reasons of drug withdrawal.

\section{Efficacy of antiretroviral therapy}

Efficacy was measured using plasma viral load, CD4+ T cell count and major clinical syndromes. The main clinical syndromes included fever persisting more than two weeks, loss of self-care ability, diarrhea more than three times per day and persisting more than one week. Through observing the syndromes, the change in clinical syndromes were divided into three categories: clinically improved (two or more clinical syndromes decreased or disappeared), no change (no change of clinical syndromes), clinical deterioration (syndromes increased).

\section{Measure of viral load and CD4+ counts}

20ml EDTA anti-coagulated whole blood was drawn and transported to the lab in an icebox. Plasma HIV-1 RNA was tested by using standardized Nulcisens method (reagent and equipment from the Co.Ltd BioMerieux). The CD4+ cells were counted by flow cytometry (Reagents and equipment from BD Company)

\section{Testing of drug resistance}

Genotypic HIV-1 drug resistance was tested with home-brew HIV-1 genotypic drug resistance methods [10, 11]. All the samples were assayed with the following algorithm: amplification of HIV-1 pol gene fragment, sequencing directly, identification of mutations related with drug resistance, analyze the mutation, explanation of drug resistance.

HIV RNA was extracted and purified from plasma with QIAamp ${ }^{\circledR}$ Viral RNA isolation kits according to the manufacturer's protocol. The HIV-1 protease and reverse transcriptase gene were amplified by Nested RT-PCR and sequenced directly. The primers were designed according to the HIV-1 subtypes circulated in China (Tab. 1).

The drug resistance analysis was based on a public database available on the Internet. In brief, sequences were submitted to the web site: http://hivdb.stanford.edu. By comparing with the sequences in the database, the software identified the drug resistance mutations and interpreted drug resistance with a scoring system in the database [12].

\section{Statistic analysis}

Comparisons among different groups were performed using $\mathrm{X}^{2}$ test and $t$ test. The $95 \%$ confidence intervals (CI) were computed using the binomial distribution.

\section{RESULTS}

\section{Study population}

138 (treated for three months) and 112 (treated for six months) AIDS patients were recruited respectively into two cross-sectional studies. All the patients were inhabit- 
ants of Shenqiu district and lived in the rural area; $95.6 \%$ of them were infected by paid plasma donation in the 1990s. 35 patients were investigated two times at three and six months of therapy. As a control, 45 antiretroviral naïve AIDS patients were investigated in the first survey.

\section{Efficacy}

The CD4+ cell count increased significantly for the patients treated for three months compared with the antiretroviral naïve patients ( $t$ Test, $\mathrm{P}<0.05$ ). However, the CD4+cell count was already decreasing in the patients treated for six months. There was no significant difference in viral load between the different groups. The alleviation rates of the main clinical syndromes following three and six month therapy were $55.1 \%$ and $50.8 \%$ respectively (Tab. 2). It is notable that the proportion of the patients who demonstrated good adherence decreased significantly from $78.8 \%$ in the three months therapy group to $33.9 \%$ in six months therapy group.

\section{Drug resistance}

Of the 45 plasma samples collected from drug naïve patients, 36 individuals were successfully amplified target gene sequences. Among them, 13.9\% variants had drugresistant mutations (Tab. 3). Compared with drug naïve group, the prevalence of drug-resistant variants increased significantly to $45.4 \%$ in three months therapy group and $62.7 \%$ in six months therapy group. Analysis of different drug-resistant mutations suggested that NNRTI-resistant variants increase significantly.

The drug resistance spectrum changed with continuation of therapy (Tab. 4). In the group treated for three months, high level drug resistance to NVP, DLV and EFV increased significantly compared to the naïve group. After treatment for six months, high level resistance to $3 \mathrm{TC}$ and FTC increased as well as DLV, EFV and NVP. At the same time, low level resistance to AZT, DDI, DDC and D4T emerged.

There were 35 patients who were tested for genotypic drug resistance two times at three and six month therapy. Compared to three months therapy, there was significant decrease in the CD4+ cell count, while there was no difference in the viral load after six months therapy. The drug-resistant mutations, especially the mutations to NRTIs increased dramatically. The most significant change was the dramatic increase in mutation frequency and sites, from 10 codons and 22 mutation times at three months therapy to 26 codons and 69 mutation times at six months therapy.

In the short-term antiretroviral therapy (within three months) group, the drug-resistant mutation sites concen-

Tab. 1 Primers of genotypic drug resistance tests

\begin{tabular}{|c|c|c|c|c|}
\hline Goal & Name & Sequence & Location & Length of amplification \\
\hline \multirow{5}{*}{ Amplification } & PR-A & 5'-CCTAGGAAAAAGGGCTGTTGGAAATGTGG & $2011-2039$ & Complete PR \\
\hline & RT-A & 5'-AACTTCTGTATGTCATTGACAGTCCA & $3303-3328$ & sequence and RT \\
\hline & RT-B & 5'-CATTTATCAGGATGGAGTTCATA & $3243-3265$ & $\mathrm{~kb}$ \\
\hline & MAW26 & 5'-TTGGAAATGTGGAAAGGAAGGAC & $2028-2050$ & Complete PR \\
\hline & RT20 & 5'-CTGCCAGTTCTAGCTCTGCTTC & $3441-3462$ & $3 \mathrm{~kb}$ \\
\hline \multirow{3}{*}{ Sequencing } & MAW46 & 5'-TCCCTCAGATCACTCTTTGGCAACGAC & $2251-2277$ & \\
\hline & RTA & 5'-GTTGACTCAGATTGGTTGCAC & $2519-2539$ & \\
\hline & RTB & 5'-CCTAGTATAAACAATGAGACAC & $2946-2967$ & \\
\hline
\end{tabular}

Tab. 2 Curative effect of antiretroviral therapy

\begin{tabular}{|c|c|c|c|c|c|c|}
\hline Groups & $\begin{array}{c}\text { CD4+ T cell } \\
\text { count }\end{array}$ & $\begin{array}{l}\text { Viral load } \\
\text { (log) }\end{array}$ & $\begin{array}{c}\text { Adherence } \\
(\%)\end{array}$ & $\begin{array}{l}\text { Clinical } \\
\text { Improve }\end{array}$ & $\begin{array}{c}\text { No } \\
\text { change }\end{array}$ & $\begin{array}{c}\text { Clinical } \\
\text { deterioration }\end{array}$ \\
\hline Drug naïve & $201.4 \pm 8.7$ & $4.8 \pm 1.5$ & - & - & - & - \\
\hline 6 mon ther & $371.7 \pm 2.2$ & $4.2 \pm 1.2$ & 33.9 & 50.8 & 40.3 & 8.9 \\
\hline
\end{tabular}


Prevalence and evolution of drug resistance HIV-1 variants in Henan, China

Tab. 3 HIV drug resistance in different groups

\begin{tabular}{|c|c|c|c|c|c|}
\hline Groups & $\begin{array}{c}\text { Overall mut } \\
(\%)\end{array}$ & $\begin{array}{c}\text { NRTI mut } \\
(\%)\end{array}$ & $\begin{array}{c}\text { NNRTI mut } \\
(\%)\end{array}$ & $\begin{array}{c}\text { PI mut } \\
(\%)\end{array}$ & $\begin{array}{l}\text { Med \& higher } \\
\text { drug Res. (\%) }\end{array}$ \\
\hline Drug naïve & 13.9 & 8.3 & 13.9 & 11.1 & 9.5 \\
\hline 6 mon ther & 62.7 & 14.8 & 47.9 & 0.0 & 61.3 \\
\hline
\end{tabular}

Tab. 4 Drug resistance spectrum

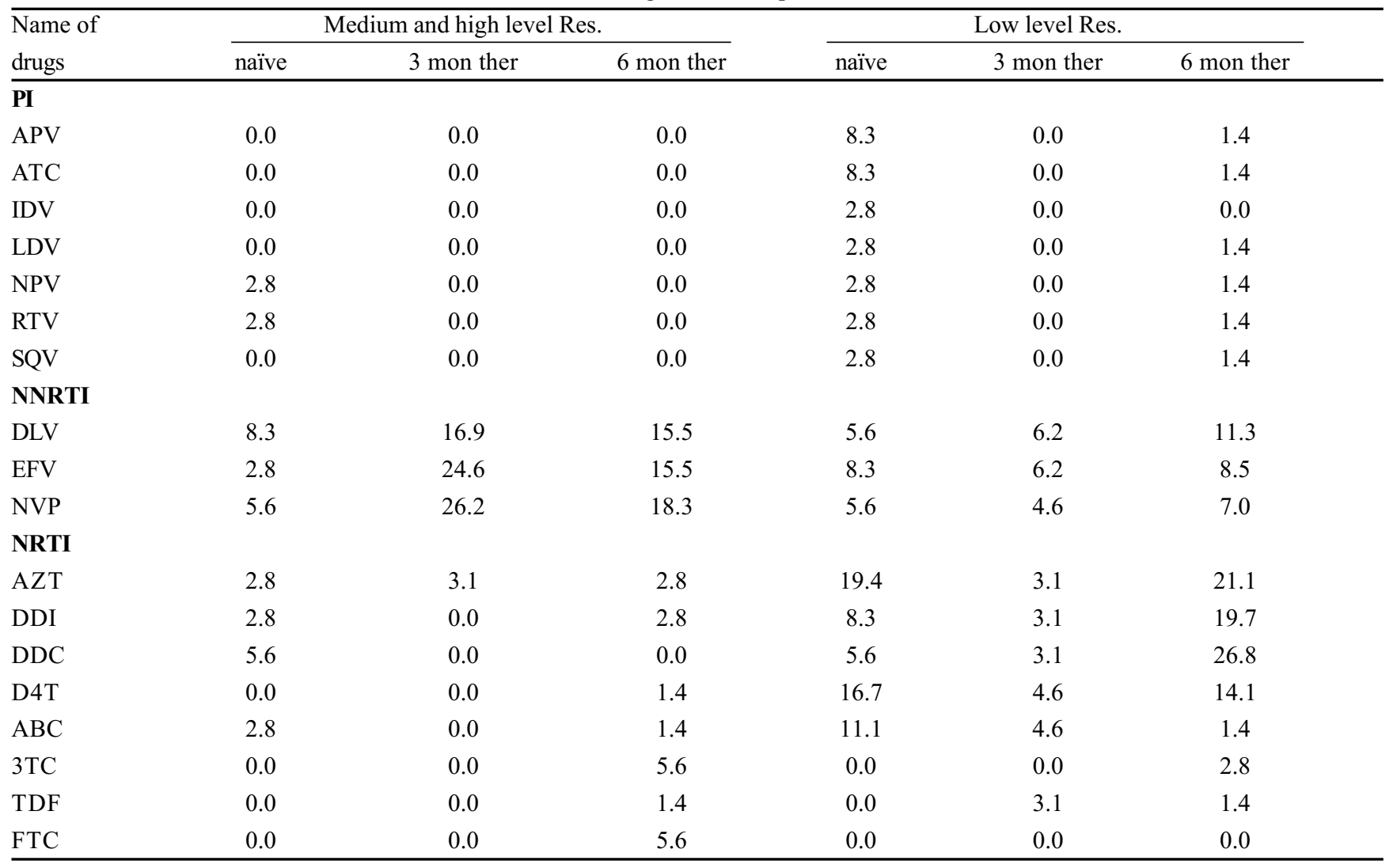

Tab. 5 Drug resistance analysis of 35 patients tested for two times

\begin{tabular}{lcc}
\hline Items & Three month therapy & Six month therapy \\
\hline CD4+T Cell Count (entry/ $\mu 1)$ & $458.4 \pm 27.9$ & $371.7 \pm 22.2$ \\
Viral load (log10) & $4.5 \pm 1.5$ & $4.2 \pm 1.2$ \\
Overall mut rate (\%) & 40.0 & 88.6 \\
NRTI Mut rate (\%) & 3.1 & 28.1 \\
NNRTI Mut rate (\%) & 25.0 & 43.8 \\
Drug resistance rate (\%) & 28.6 & 34.3 \\
Mut codon/mut frequency & $10 / 22$ & $26 / 69$ \\
First 5 mut codon & $103,106,318,215,109$ & $106,67,151,190,236$ \\
\hline
\end{tabular}


Tab.6 Frequency and site of drug-resistant mutation

\begin{tabular}{|c|c|c|c|}
\hline \multirow[t]{2}{*}{ Mutation } & \multicolumn{2}{|c|}{ Frequency of mutation } & \multirow[t]{2}{*}{ Drug Resistance explanation } \\
\hline & 3 mon ther & 6 mon ther & \\
\hline E44K & $0(0.0)$ & $3(8.7)$ & Potential resistance to NERI \\
\hline D67N & $0(0.0)$ & $6(17.4)$ & NRTI resistance except for AZT,FTC \\
\hline T69G & $0(0.0)$ & $1(2.9)$ & Low resistance to DDI \\
\hline K103N & $6(17.1)$ & $2(5.7)$ & High resistance to NNRTI \\
\hline V106I/L & $4(11.4)$ & $6(17.4)$ & $\begin{array}{l}\text { High resistance to NVP and EFV, } \\
\text { Medium resistance to DLV }\end{array}$ \\
\hline V108F/A & $0(0.0)$ & $2(5.7)$ & Potential resistance to D4T and DDI \\
\hline Y181C & $1(2.9)$ & $2(5.7)$ & $\begin{array}{l}\text { High resistance to NVP and DLV, low } \\
\text { resistance to EFV }\end{array}$ \\
\hline M184I & $0(0.0)$ & $2(5.7)$ & High resistance to $3 \mathrm{TC}$ and $\mathrm{FTC}$ \\
\hline G190A & $2(5.7)$ & $4(11.6)$ & $\begin{array}{l}\text { High resistance to NVP and EFV, Medium } \\
\text { resistance to DLV }\end{array}$ \\
\hline $\mathrm{L} 210 \mathrm{C} / \mathrm{F}$ & $1(2.9)$ & $3(8.7)$ & $\begin{array}{l}\text { No typical mutation or NRTI resistance } \\
\text { except for } 3 \text { TC,FTC }\end{array}$ \\
\hline $\mathrm{T} 215 \mathrm{~S}$ & $2(5.7)$ & $1(2.9)$ & Low resistance to $\mathrm{AZT}$ and $\mathrm{D} 4 \mathrm{~T}$ \\
\hline
\end{tabular}

trate in codons 103, 106 and 215, two of which induce high level resistance to NNRTIs. After longer-term antiretroviral therapy, the six months group, the number of drug-resistant mutation codons increased significantly. Among them, 44, 65, 75, 103, 106, 108, 118, 151, 190, $210,225,227,230,236,238$ site mutations occurred in more than $5 \%$ samples. These mutation sites can cause low level drug resistance to NRTIs and high level resistance to NNRTIs (Tab. 5).

\section{DISCUSSIONS AND CONCLUSIONS}

The drug resistance mutations of protease can be divided into primary mutations and secondary mutations. Primary mutations are those that reduce drug susceptibility by themselves whereas secondary mutations reduce drug susceptibility in combination with primary mutations or improve the replicative fitness of virus isolates with a primary mutation [13]. The mutations conferring resistance to reverse transcriptase inhibitors can be relatively simple. A single point mutation can induce high level resistance to all of the NNRTIs, i.e., cross-resistance [14, 15]. The targets of NRTIs and NNRTIs are different. When converted into the triphosphate forms by phosphorylation, NRTIs compete with natural triphosphate, incorporate into the DNA chain, and lead to the termination of viral DNA synthesis [16]. NNRTIs are a series compounds with multiple structures, which by binding to the hydrophobic region near the active sites of reverse transcriptase inhibit the activity of the enzyme [17]. The NRTIs all bind to the same site of RT, and due to the decline of binding stability 
or stereospecific blockade, susceptibility to NRTIs is reduced.

Since there were no protease inhibitors in the antiretroviral therapy regimen in Henan, it is not surprising that we didn't find resistance to protease inhibitors. Because RTI (reverse transcriptase inhibitors)-resistant mutations can be simple, some single point mutations can induce high level resistance to many of the RTIs, and induce the cross-resistance. In our research, the 103, 106, 109,230 and 238 codons in the reverse transcriptase gene are the most apt sites to be mutated. These mutations emerge shortly and quickly after the beginning of combination therapy. As a result, the high level resistance to NVP, DLV and EFV occurred in the patients.

The prevalence rate of HIV drug-resistant variants in drug naïve patients was $13.1 \%$, lower than that in Europe and the U.S., suggesting that drug-resistant HIV variants were not prevalent before the implementation of antiretroviral therapy in Henan. After treatment with antiretroviral medicines, the viral variants are under the drugs' selective pressure. Most of the susceptible variants are killed; the other variants replicate quickly and overgrow the susceptible variants. In our research, the NNRTIresistant mutations occurred first and quickly. The prevalence rate increased from $3.3 \%$ in the naïve therapy group to $39.5 \%$ in the three months therapy group and $47.9 \%$ in the six months therapy group. Compared to NNRTI-resistant mutations, NRTI-resistant mutations emerged later and more slowly. NRTI resistance mutations were found in $8.3 \%$ of drug naïve patients, $11.9 \%$ of the three months therapy patients and $14.8 \%$ of the six months therapy patients respectively. This was probably due to the different mechanisms of drug-resistance for NRTIs and NNRTIs. For NNRTIs, single point mutations can induce high level resistance and cross-resistance, whereas more mutations are needed to induce the resistance to some NRTIs such as ZDV.

With the implementation of free antiretroviral therapy in this study, the main clinical syndromes were alleviated; the CD4+ cell count increased in the three month treatment patients, whereas the viral load did not change significantly. This was probably due to the poor adherence noted in these groups of patients. Adherence is one of the most important factors that influence the clinical improvement and drug resistance. Successful treatment of HIV/AIDS requires $95 \%$ or greater adherence to highly active antiretroviral therapy [13]. However, 66\% patients involved in our research did not have good adherence after six months therapy. The reasons for non-adherence included side effects and grow out of taking medicines. Maintaining good adherence is a great challenge in the future of antiretroviral therapy in China. With the emergence and prevalence of drug-resistant variants, the risk of drug resistance variants transmission increased. Anyway, it's necessary to improve the adherence of patients in the free therapy program. Measures should be taken to educate patients, simplify dosing and enhance adherence monitoring. Consideration should even be given to directly observed therapy (DOT) [18].

\section{ACKNOWLEDGEMENTS}

This work was supported by the Grants from Ministry of Health and the National HIV drug Resistance Surveillance Network of China.

\section{REFERENCES}

1 Mansky LM. Retrovirus mutation rates and their role in genetic variation. J Gen Virol 1998; 79:1337-45.

2 Coffin JM. HIV population dynamics in vivo: implications for genetic variation, pathogenesis, and therapy. Science 1995; 267: 483-9.

3 Droz C, Morand-Joubert L, Raguin G, et al. Impact and evolution of resistance in patients treated by a salvage regimen combining amprenavir, lopinavir and ritonavir (the puzzle1 study). Antiviral Ther 2002; 7(Suppl 1):S111.

4 Little SJ, Holte S, Routy JP, et al. Antiretroviral drug resistance among patients recently infected with HIV. N Eng J Med 2002; 347:385-94.

5 Wensing A, Vijver Dvd, Asjo B, et al. Prevalence of transmitted drug resistance in Europe is largely influenced by the presence of non-B sequences: analysis of 1400 patients from 16 countries: the CATCH-Study. Antivir Ther 2003; 8:S131.

6 Bennett DE, Zaidi I, Heneine W, et al. Prevalence of mutations associated with antiretroviral drug resistance among men and women newly diagnosed with HIV in 10 US cities, 1997-2001. Antivir Ther 2003; 8:S133.

7 Chaix M, Descamps D, Mouajjah S, et al. Resistance Group Cohort PRIMO INTERPRIM and PRIMSTOP Study Groups. French National Sentinel Survey of antiretroviral resistance in patients with HIV-1 primary infection and in antiretroviral-naive chronically infected patients in 2001-2002. Antivir Ther 2003; 8:S137.

8 DeGruttola V, Dix L, D.Aquila $\mathrm{R} £ \neg$ et al. The relation between baseline HIV drug resistance and response to antiretroviral therapy: re-analysis of retrospective and prospective studies using a standardized data analysis plan. Antivir Ther 2000; 5:418.

9 Haubrich R, Demeter L. Clinical utility of resistance testing: retrospective and prospective data supporting use and current recommendations. J Acquir Immune Defic Syndr 2001; 26 (Suppl 1): S51-9.

10 Duraut J, Clevenbergh P, Halfon P, et al. Drug-resistance genotyping in HIV-1 therapy, The VIRADAPT randomized controlled trial. Lancet 1999; 353:2195-9.

11 Daxter JD, Mayers DL, Wantworth DN, et al. A randomized study of antiretroviral management based on plasma genotypic antiretroviral resistance in patients failing therapy. CPCRA 046 study team for the Terry Beirn Community Programs for Clinical Research on AIDS. AIDS 2000; 14:F83-93. 
12 Rhee SY, Gonzales MJ, Kantor R, et al. Human immunodeficiency virus reverse transcriptase and protease sequence database. Nucleic Acids Res 2003; 31:298-303.

13 Shafer RW. Genotypic Testing for HIV-1 Drug Resistance. HIV Insite Knowledge Base Chapter, April 2004. http://www. hivinsite.com

14 Havlir DV, Eastman S, Gamst A, et al. Nevirapine resistant human immunodeficiency virus: kinetics of replication and estimated prevalence in untreated patients. J Virol 1996; 70:7894-9.

15 Conway B, Weinberg MA, Hall D, et al. Development of drug resistance in patients receving combinations of zidovudine, didanosine and nevirapine. AIDS 2001; 15:1269-74.

16 Kohlstaedt LA, Wang J, Friedman JM, et al. Crystal structure at 3.5 A resolution of HIV-1 reverse transcriptase complexed with an inhibitor. Science 1992; 256:1783-90.

17 Esnouf R, Ren J, Ross C, et al. Mechanism of inhibition of HIV1 reverse transcriptase by non-nucleoside inhibitors. Nat Struct Biol 1995; 2:303-8.

18 Cingolani A, Antinori A, Rizzo MG, et al. Usefulness of monitoring HIV drug resistance and adherence in individuals failing highly active antiretroviral therapy: a randomized study (ARGENTA). AIDS 2002; 16:369-79. 